\title{
УСЛОВИЯ, ПРИ КОТОРЫХ КОРНИ МНОГОЧЛЕНА ЛЕЖАТ ВНУТРИ ЕДИНИЧНОГО КРУГА
}

\author{
В. Г. Задорожний \\ Воронежский государственный университет
}

Поступила в редакцию 06.04.2018 г.

\begin{abstract}
Аннотация. Для решения дифференциальных уравнений разностными методами требуется исследовать разностную схему на устойчивость. Если корни соответствующего линейного оператора по модулю меньше единицы то схема устойчива. В статье проблема нахождения условий, при выполнении которых модули всех корней многочлена меньше единицы, сводится к проверке условий Рауса - Гурвица для специального многочлена, который строится по исходной задаче. Приведены коэффициентные условия для многочленов второго и третьего порядков. Метод удобен при теоретическом исследовании систем, можно указать области изменения коэффициентов, при которых сохраняется свойство принадлежности корней единичному кругу.

Ключевые слова: корни многочлена, признак Рауса - Гурвица, корни в единичном круге, спектральный радиус.

Annotation. For solution of differential equations with difference methods required to investigate difference scheme on stability. If the modulo of roots of the corresponding linear operator is less than one then the scheme is stability. In the article the problem of finding the conditions under which all modules of the roots of a polynomial is less than one, reduced to Routh-Hurwitz conditions for special polynomial, which is built on the original problem. Coefficient conditions for polynomials of the second and third orders are given. Method is useful when the theoretical study systems, you can specify coefficients change, when storing the property conditioning roots a single circle.

Keywords: the roots of the polynomial, the sign of the Routh - Hurwitz, the roots in the unit circle, the spectral radius.
\end{abstract}

\section{1. ВВЕДЕНИЕ И ПОСТАНОВКА ЗАДАЧИ}

При решении дифференциальных уравнений разностными методами изучается устойчивость метода. При этом выписывается соответствующий данному методу линейный оператор, и находятся условия, при которых спектральный радиус оператора не превосходит единицу. Если оператор действует в конечномерном пространстве, то можно выписать матрицу линейного оператора и найти ее характеристический многочлен. Если корни характеристического многочлена по модулю меньше единицы, то спектральный радиус матрицы также меньше единицы.

Задача нахождения удобных условий, при выполнении которых корни многочлена располагаются внутри единичного круга, встре-

( ) Задорожний В. Г., 2018 чается очень часто. Например, требуется решить линейную неоднородную алгебраическую систему уравнений $A x=b$, где $A$ - линейный ограниченный оператор, действующий в $n$-мерном пространстве, $x$ - искомый вектор, $b$ - заданный вектор. Уравнение можно записать в виде $x=B x+b$, где $B=E-A$, $E$ - единичный оператор. Если спектральный радиус оператора $B$ меньше единицы, то отображение $B x+b$ является сжимающим и решение может быть получено методом простой итерации [1, стр. 335, 2, стр. 218] как предел последовательности $\left\{x_{k}\right\}$, где $x_{k}=B x_{k-1}+b$, $k=1,2, \ldots$, причем начальный вектор $x_{0}$ можно выбирать произвольным образом. Если собственные значения оператора $B$ лежат внутри единичного круга, то спектральный радиус [3, стр. 252] оператора $B$ меньше единицы. Собственные значения оператора $B$ 
являются корнями характеристического многочлена $\operatorname{det}(B-z E)$.

При этом естественной является задача: Найти условия, при которых модули всех корней многочлена

$$
p=a_{0} z^{n}+a_{1} z^{n-1}+\cdots+a_{n}, a_{0}>0
$$

меньше единицы.

По этой причине уместно следующее

Определение. Многочлен $p$ называется многочленом сжатия, если все его корни лежат внутри единичного круга.

Эта задача возникает при решении дифференциальных уравнений с периодическими коэффициентами. Если все мультипликаторы линейной системы дифференциальных уравнений с периодическими коэффициентами по модулю меньше единицы, то система асимптотически устойчива [4, стр. 89]. Однако для решения задачи указываются методы, связанные с нахождением спектрального радиуса оператора $[5,6]$. В статье Г. Корсакова [7] приводится способ решения этой задачи, но метод не приводит к правильным результатам.

В постановке задачи бросается в глаза аналогия с задачей об условиях устойчивости многочлена $[8,9]$ : найти условия, при которых вещественные части всех корней многочлена меньше нуля. Решение последней задачи общеизвестно и дается, например, признаком Рауса - Гурвица $[8,9]$.

\section{2. СВЕДЕНИЕ К ЗАДАЧЕ ОБ УСТОЙЧИВОСТИ МНОГОЧЛЕНА}

Теорема 1. Для того чтобы модули всех коней многочлена (1) были меньше единицы, необходимо и достаточно, чтобы вещественные части всех корней многочлена

$$
\begin{aligned}
& q(\zeta)=a_{0}(\zeta+1)^{n}+a_{1}(\zeta+1)^{n-1}(\zeta-1)+ \\
& +a_{2}(\zeta+1)^{n-2}(\zeta-1)^{2}+\cdots+a_{n}(\zeta-1)^{n}
\end{aligned}
$$

были отрицательны (т. е. чтобы многочлен $q$ был устойчив).

Доказательство. Сделаем дробно-линейную замену переменной

$$
z=\frac{\zeta+1}{\zeta-1}
$$

При этом числу $z=i$ соответствует $\zeta=-i$, числу $z=-1$ соответствует $\zeta=0$ и числу $z=-i$ соответствует число $\zeta=i$. При дробно-линейном преобразовании окружности отображаются либо в окружности, либо в прямые линии [10, стр. 216]. Тогда единичная окружность плоскости переменной $z$ отображается в мнимую ось плоскости переменной $\zeta$, а внутренность единичного круга отображается в левую полуплоскость от мнимой оси переменной $\zeta$. При этом

$$
p\left(\frac{\zeta+1}{\zeta-1}\right)=a_{0}\left(\frac{\zeta+1}{\zeta-1}\right)^{n}+a_{1}\left(\frac{\zeta+1}{\zeta-1}\right)^{n-1}+\cdots+a_{n}
$$

и корни многочлена $p$ отображаются в корни уравнения

$$
\begin{gathered}
a_{0}(\zeta+1)^{n}+a_{1}(\zeta+1)^{n-1}(\zeta-1)+ \\
+a_{2}(\zeta+1)^{n-2}(\zeta-1)^{2}+\cdots+a_{n}(\zeta-1)^{n}=0 .
\end{gathered}
$$

Теорема доказана.

Таким образом, чтобы выяснить является ли $p$ многочленом сжатия, нужно выписать многочлен $q$, преобразовать его к стандартному виду по степеням $\zeta$ и применить признак Рауса - Гурвица. Можно использовать и другие признаки устойчивости многочлена (2), например, признак Михайлова - Найквиста [5, стр. 123], признак Попова [5] и др.

\section{3. МНОГОЧЛЕНЫ ПЕРВОЙ И ВТОРОЙ СТЕПЕНИ}

Рассмотрим многочлен первой степени $p_{1}(z)=a_{0} z+a_{1}$. Очевидно, он является многочленом сжатия при выполнении условия $\left|a_{1}\right|<\left|a_{0}\right|$.

Теорема 2. Для того чтобы вещественный многочлен

$$
p_{2}(z)=z^{2}+a_{1} z+a_{2}
$$

был многочленом сжатия, необходимо и достаточно, чтобы выполнялись условия

$$
1+a_{1}+a_{2}>0,1-a_{2}>0,1-a_{1}+a_{2}>0 .
$$

Доказательство. Выпишем многочлен

$$
\begin{aligned}
& q(\zeta)=(\zeta+1)^{2}+a_{1}(\zeta+1)(\zeta-1)+a_{2}(\zeta-1)^{2}= \\
& =\left(1+a_{1}+a_{2}\right) \zeta^{2}+2\left(1-a_{2}\right) \zeta+1-a_{1}+a_{2} .
\end{aligned}
$$

Из условий Рауса - Гурвица следует, что вещественный многочлен второй степени устойчив, если и только если, его коэффици- 


\section{В. Г. Задорожний}

енты положительны. Следовательно, выполняются условия (3). Теорема доказана.

Условия (3) означают, что точки $M\left(a \_1, a \_2\right)$ лежат внутри треугольника АВC (см. рис. 1).

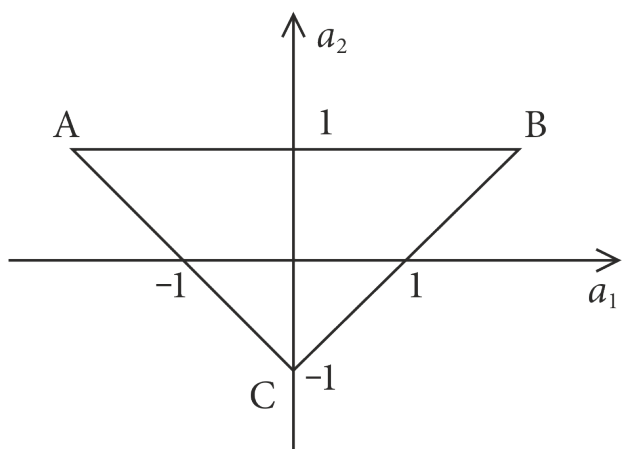

Puc. 1

\section{4. МНОГОЧЛЕН ТРЕТЬЕЙ СТЕПЕНИ}

Теорема 3. Для того чтобы вещественный многочлен

$$
p_{3}(z)=z^{3}+a_{1} z^{2}+a_{2} z+a_{3}
$$

был многочленом сжатия, необходимо и достаточно, чтобы выполнялись условия

$$
\begin{gathered}
1+a_{1}+a_{2}+a_{3}>0, \\
3\left(1+a_{3}\right)-\left(a_{1}+a_{2}\right)>0, \\
1-a_{1}+a_{2}-a_{3}>0, \\
a_{2}^{2}-4 a_{1} a_{3}+4 a_{2}-1<0 .
\end{gathered}
$$

Доказательство. Выпишем многочлен

$$
\begin{gathered}
q(\zeta)=(\zeta+1)^{3}+a_{1}(\zeta+1)^{2}(\zeta-1)+ \\
+a_{2}(\zeta+1)(\zeta-1)^{2}+a_{3}(\zeta-1)^{3}= \\
=\left(1+a_{1}+a_{2}+a_{3}\right) \zeta^{3}+\left(3\left(1-a_{3}\right)+a_{1}-a_{2}\right) \zeta^{2}+ \\
+\left(3-a_{1}-a_{2}+3 a_{3}\right) \zeta+1-a_{1}+a_{2}-a_{3} .
\end{gathered}
$$

Условия Рауса - Гурвица для этого многочлена имеют вид

$$
\begin{gathered}
1+a_{1}+a_{2}+a_{3}>0, \\
3\left(1-a_{3}\right)+a_{1}-a_{2}>0, \\
3\left(1+a_{3}\right)-\left(a_{1}+a_{2}\right)>0, \\
1-a_{1}+a_{2}-a_{3}>0, \\
{\left[3\left(1-a_{3}\right)+a_{1}-a_{2}\right]\left[3\left(1+a_{3}\right)-\left(a_{1}+a_{2}\right)\right]>} \\
>\left[1-a_{1}+a_{2}-a_{3}\right]\left[1+a_{1}+a_{2}+a_{3}\right] .
\end{gathered}
$$

Легко видеть, что второе неравенство является следствием остальных неравенств и его можно не учитывать. Последнее неравенство преобразуется к виду $a_{2}^{2}-4 a_{1} a_{3}+4 a_{2}-1<0$. Теорема доказана.

\section{5. ЗАКЛЮЧЕНИЕ}

Мы рассмотрели вещественные многочлены второго и третьего порядков, которые чаще встречаются в приложениях, однако метод применим и для многочленов с комплексными коэффициентами, но при этом комплексным является и многочлен $q$. Отметим, что число корней многочлена $p$, лежащих внутри единичного круга, равно числу корней многочлена $q$, лежащих слева от мнимой оси. В настоящее время имеются компьютерные программы, которые эффективно находят спектры матриц. Однако для этого коэффициенты матриц должны быть числами. Предложенный метод удобен для аналитических исследований систем. При этом можно найти области изменения параметров системы, при которых сохраняется устойчивость.

\section{СПИСОК ЛИТЕРАТУРЫ}

1. Бахвалов Н. С. Численные методы / Н. С. Бахвалов. - М. : Наука, 1975. - 631 с.

2. Коллати, Л. Функциональный анализ и вычислительная математика / Л. Коллатц. М. : Мир, 1969. - 467 с.

3. Колмогоров А. Н. Элементы теории функций и функционального анализа /А. Н. Колмогоров, С. В. Фомин. - М. : Физматлит, 2004. - 570 c.

4. Якубович В. А. Линейные дифференциальные уравнения с периодическими коэффициентами / В. А. Якубович, В. М. Старжинский. - М. : Наука, 1972. - 718 с.

5. Справочник по теории автоматического управления. - М. : Наука, 1987. - 711 с.

6. Беллман Р. Введение в теорию матриц / Р. Беллман. - М. : Наука, 1969. - 367 с.

7. Корсаков Г. Ф. О количестве корней полинома вне круга / Г. Ф. Корсаков // Математические заметки. - Т. 13, вып.1. - 1973. C. $3-12$.

8. Гантмахер Ф. Р. Теория матриц / Ф. Р. Гантмахер. - М. : Наука, 1967. 
9. Постников М. М. Устойчивые многочлены / М. М. Постников. - М. : Наука, 1981. $176 \mathrm{c}$.

Задорожний Владимир Григорьевич - д-р физ.-мат. наук, профессор, зав. кафедрой системного анализа и управления, Воронежский государственный университет.

Тел.: 8-473-274-14-85

E-mail: zador@amm.vsu.ru
10. Корн Г. Справочник по математике / Г. Корн, Т. Корн. - М. : Наука, 1984. - 831 с.

Zadorozhniy Vladimir Grigoryevich - doctor of science, professor, Department of system analysis and control, Voronezh State University.

Tel.: 8-473-274-14-85

E-mail: zador@amm.vsu.ru 